\title{
LES SITUATIONS DE GESTION : ENTRE INTENTIONNALITÉ ET PROBLÉMATISATION
}

\author{
$\underline{\text { Christophe Schmitt }}$
}

De Boeck Supérieur | « Projectics / Proyéctica / Projectique »

$2017 / 2 \mathrm{n}^{\circ} 17$ | pages 9 à 24

ISSN 2031-9703

ISBN 9782807391314

Article disponible en ligne à l'adresse :

https:/www.cairn.info/revue-projectique-2017-2-page-9.htm

Distribution électronique Cairn.info pour De Boeck Supérieur.

(C) De Boeck Supérieur. Tous droits réservés pour tous pays.

La reproduction ou représentation de cet article, notamment par photocopie, n'est autorisée que dans les limites des conditions générales d'utilisation du site ou, le cas échéant, des conditions générales de la licence souscrite par votre établissement. Toute autre reproduction ou représentation, en tout ou partie, sous quelque forme et de quelque manière que ce soit, est interdite sauf accord préalable et écrit de l'éditeur, en dehors des cas prévus par la législation en vigueur en France. Il est précisé que son stockage dans une base de données est également interdit. 


\section{LES SITUATIONS DE GESTION : ENTRE INTENTIONNALITÉ ET PROBLÉMATISATION ${ }^{1}$}

\section{MANAGEMENT SITUATIONS: BETWEEN INTENTIONALITY AND PROBLEMATIZATION}

\section{Christophe Schmitt}

Professeur et Titulaire de la Chaire Entreprendre, IAE de Metz Université de Lorraine

\section{RÉSUMÉ}

La notion de situation est étonnamment très peu mobilisée en management. L'objectif de cet article est de s'interroger sur la pertinence de cette notion pour comprendre la complexité des organisations. Nous montrons aussi que cette notion permet d'envisager les différentes interactions entre les acteurs, leur intentionnalité ou encore la dynamique de ces interactions. Au final, nous proposons une définition de la notion de situation de gestion.

Mots-clés : situation de gestion, intentionnalité, problématisation, complexité, interaction

\section{ABSTRACT}

The notion of situation is surprisingly very little involved in management. The purpose of this article is to question the relevance of this notion in order to understand the complexity of organizations. We will also show that this notion helps consider the different interactions between the actors, their intentionality or the dynamics of these interactions. Finally, we propose a definition of the notion of management situation.

Keywords: management situation, intentionality, problematization, complexity; interaction

1. Une première version de cet article a fait l'objet d'une publication sous le titre Aborder la complexité des organisations par les situations de gestion, dans SCHMITT C., De la complexité de l'action dans les organisations, Growth Publisher, p. 13-29. 
Etrangement, la notion de situation s'est largement développée dans le domaine des sciences humaines sans toutefois réussir à percer au niveau du management. Pourtant cette notion est très intéressante pour aborder la complexité d'une situation, notamment les différentes interactions entre les acteurs de la situation, leur incertitude ou encore leur dynamique. Peu présentes dans les recherches en sciences de gestion en général, les situations ont fait l'objet de beaucoup de travaux dans les domaines aussi variés que « la sémantique, la logique, l'anthropologie cognitive, l'intelligence artificielle, la psychologie écologique, et dans différentes entreprises se réclamant du paradigme de 'l'action située’ » (de Fornel et Quéré, 1999). Or, les différents travaux portant sur la notion de gestion en management amènent des perspectives intéressantes pour aborder la complexité des organisations (Girin, 1990 ; Journé et Raulet-Croset, 2008 ; Schmitt, 2009 ; Schmitt et al., 2011 ; Schmitt, 2015). À travers cette notion, il ne s'agit pas de mettre simplement au centre de la réflexion une notion trop longtemps négligée, mais d'élaborer une nouvelle conception du management et de l'intelligence humaine en situation, en redonnant toute son importance à la dynamique des interactions entre le manager et son environnement dans une perspective phénoménologique, c'est-à-dire basée sur l'expérience vécue par le manager en situation. Recourir à cette notion n'est pas neutre pour la recherche en management. En effet, elle interpelle nos connaissances et interroge notre posture épistémologique et méthodologique en tant que chercheur. Le but de cet article est justement de tracer les contours de cette notion de situation de gestion.

Le présent article se divise en deux parties. La première partie aborde la place des situations de gestion dans la recherche en management. Force est de constater la faible présence de cette notion dans les réflexions portant sur le management. Plus précisément, il est possible de qualifier d'absente cette notion de façon implicite. Elle ne fait pas partie des thèmes de recherche susceptibles de se profiler au travers de la recherche en management. C'est situation est paradoxale, car la notion de situation est souvent évoquée dans les travaux de recherche sans pour autant être considérée comme un objet de recherche à part entière ; pis encore, la notion est mobilisée mais rarement définie. Ainsi insistons-nous particulièrement sur les limites de ces approches pour comprendre la complexité des organisations en management et, plus particulièrement, des pratiques managériales.

À partir de ces éléments, la seconde partie de l'article s'intéresse à la caractérisation de la notion de situation de gestion. Dans le premier point, nous introduisons une notion importante pour comprendre une situation de gestion : l'intentionnalité. Nous nous appuyons sur les réflexions menées dans le domaine de la phénoménologie pour étayer la notion de situation de gestion. Le deuxième point introduit une notion supplémentaire : l'espace de problématisation. La position que nous défendons dans cet article est que l'espace de problématisation joue un rôle dans la structuration des situations de gestion. Ainsi, manager n'est pas une activité coupée de tout, mais elle est plutôt cognitive et s'inscrit dans une relation temporelle prenant en considération la finalité et les moyens. Enfin, dans le troisième point de cette partie nous proposons une définition des situations de gestion et des avenues de recherche pour la communauté en management. 


\section{LA PLACE DES SITUATIONS DE GESTION EN MANAGEMENT : UNE ABSENCE COMPRÉHENSIBLE}

Alors que le concept de situation de gestion a fait l'objet de nombreux développements dans différents domaines en sciences humaines et sociales (de Fornel et Quéré, 1999) comme la sociologie, la linguistique, l'anthropologie, etc., les travaux abordant explicitement ce concept en management sont plutôt rares. Pourtant, les situations de gestion semblent permettre de renouveler le regard apporté par le chercheur pour comprendre la complexité des pratiques managériales (Girin, 1990 et 2011 ; Journé et Raulet-Croset, 2008 ; Schmitt, 2009 ; Schmitt et al., 2011).

Plus précisément, la notion de situation de gestion apparaît explicitement en management, notamment à travers les travaux de Girin (1990). Toutefois, recourir à la notion de situation de gestion n'est pas neutre pour la recherche portant sur les organisations (Schmitt, 2009). Elle renvoie à des questionnements épistémologiques, méthodologiques et paradigmatiques autour des pratiques managériales. Plus précisément, le recours aux situations de gestion comme grille d'analyse de la complexité des pratiques managériales invite à s'affranchir de l'individualisme méthodologique dans lequel les recherches en gestion se sont largement inscrites. Cette partie se propose plus particulièrement de s'interroger sur la place du concept de situations de gestion dans les pratiques managériales.

\section{Le concept de situation de gestion en management}

En se construisant, soit autour de l'acteur, soit autour de ses actions, la recherche en management a longtemps négligé d'envisager les pratiques managériales à travers l'action et, notamment, à travers l'action située². Il faut attendre les travaux de Girin en 1990 pour voir ce concept apparaître au niveau de la recherche en gestion. Selon cet auteur, on parlerait d'une situation de gestion «lorsque des participants sont réunis et doivent accomplir dans un temps déterminé, une action collective conduisant à un résultat soumis à un jugement externe » (Girin, 1990). Cette définition intéressante demande néanmoins à être précisée et complétée.

En effet, elle renvoie avant tout à la problématique qui a longtemps prévalu en sciences sociales autour de l'idée de « définition de la situation » (de Fornel et Quéré, 1999) : pour agir efficacement, un acteur doit sélectionner

\footnotetext{
2. Cette notion peut se retrouver dans les recherches portant sur les processus de gestion. Mais, dans ce cas de figure, la notion de situation est souvent comprise comme centrée sur 'l'objet de recherche' négligeant l'acteur luimême. En effet, cette approche par les situations consiste à analyser l'action elle-même et ses composantes en la séparant de la personne en situation. Il s'agit d'une réflexion sur l'action alors que nous proposons, dans la perspective des sciences de l'artificiel, une réflexion dans l'action.
} 
et analyser les conditions de son action. Cette définition s'inscrit dans une rationalité et une optimisation de l'action. La définition proposée combine une inspiration kantienne (présupposition d'un sujet tenu pour source du sens et origines de ses actes) et une inspiration pragmatiste (la validité des idées et des propositions est évaluée en fonction de leurs conséquences). Une des difficultés provient de la volonté d'abstraire l'action de ses circonstances. De façon générale, comme le souligne Suchman (1987), « mieux vaut étudier comment [les gestionnaires] utilisent leurs circonstances pour effectuer une action intelligente ».

Il convient donc d'introduire, aux côtés mêmes du concept de situation de gestion, la notion d'action «située » pour évoquer la prise en compte de circonstances liées aux situations. En ce sens, le comportement des agents ne découle plus directement de l'agent mais de l'interaction avec son environnement. Cela sous-entend que chaque acteur de la situation de gestion interagit avec son environnement (autres acteurs et/ou objets dans cet environnement) et donc que des représentations différentes vont interagir dans la situation de gestion. De plus, les avancées dans le domaine de la théorie des situations (Béguin et Clot, 2004) montrent que les problématiques traditionnelles ne permettent pas de rendre compte de la logique effective (Schmitt, 2009), autrement dit de ce que fait l'acteur en situation, ni des formes de contrôle que celle-là exerce sur l'expérience. Comme Journé et Raulet-Croset (2008), nous soutenons que le concept de situation permet de rendre compte du fait que les pratiques managériales se construisent dans la complexité de l'action (Hatchuel, 2005 ; Lorino, 2005).

\section{L'action managériale : le parent pauvre de la recherche en management}

En effet, même si l'action est omniprésente dans la recherche en management, peu de travaux portent sur une contribution à une théorie de l'action y afférente (Hatchuel, 2005 ; Lorino, 2005). Cette question de la place de l'action dans la recherche en management semble importante pour la poursuite du débat et du développement de la connaissance dans le domaine de la gestion. Plus précisément, la discussion qui suit se place principalement sur le terrain des liens entre connaissance et action dans le domaine du management. II s'agit de sortir de la posture historique où la connaissance s'est construite sur une hypothèse implicite de séparation de l'action et du manager d'un côté, en l'occurrence, l'agir rationnel centré sur le porteur de l'action, le manager, et, de l'autre, l'agir normatif, renvoyant au résultat de l'action, celle du manager (Schmitt, 2015). La question sous-jacente à ces nouvelles voies de recherche est la suivante : «Viennent-elles renforcer la dichotomie de l'action ou permettre de la dépasser ? »Certaines voies de recherche essaient de dépasser cette dichotomie, s'il en est une, voire de s'en affranchir. L'enjeu derrière tout cela n'est pas neutre : il s'agit de décloisonner la recherche en management. Ce propos pourrait étonner le lecteur. En effet, la recherche en management serait-elle cloisonnée ? La recherche en management est récente en comparaison avec la recherche dans d'autres domaines et elle s'est construite 
à partir de différentes disciplines, en l'occurrence l'économie, le droit, la gestion, la sociologie, la psychologie... Aujourd'hui, la recherche en management, en tant que discipline à part entière, crée légitimement des barrières par rapport aux autres disciplines. Mais l'érection de ces barrières risque de graver dans le marbre la dichotomie de l'action évoquée dans cet article et d'empêcher la recherche en management de s'engager dans d'autres voies, dont celle de la théorie de l'action. Au final, le constat que nous pouvons dresser de la recherche dans le domaine managérial menée jusqu'ici pourrait bien s'énoncer de la manière suivante: l'action reste relativement absente du débat scientifique dans le domaine du management. Il conviendrait donc de mettre l'action au centre de la réflexion (Lorino, 2005 ; Schmitt, 2015).

\section{La recherche en gestion : un cadre convergent mais limités}

En somme, il est possible d'avancer que les recherches en management autour des pratiques managériales cherchent avant tout à rendre objectivable la réalité du manager, qu'elle soit effectivement objective ou subjective. Deux aspects de la recherche en management peuvent être mis en évidence par rapport à cette volonté d'objectivation : la prégnance d'un réel unique dans l'agir cognitif et de l'individualisme méthodologique.

Les travaux portant sur les pratiques managériales semblent demeurer attachés à une tradition de recherche où l'individu, ici le manager, est envisagé dans une réalité ontologique qui renvoie à l'idée d'enquête sur la nature des choses, de l'être, de l'existence (Lawson, 1996). Une confusion implicite existe sur le plan de la recherche en management. À travers la réalité et, notamment, à travers ce qui est apparent, il s'agit avant tout de montrer un réalisme qui cherche à découvrir les régularités empiriques du monde. Le paradigme épistémologique retenu par le chercheur, qu'il soit explicite ou non dans ses travaux, influe sur le type de recherche menée, par exemple les thèmes et questions de recherche étudiés, ainsi que sur les méthodologies employées (Burrell et Morgan, 1979 ; Popper, 1991, 1997 ; Van de Ven, 2007). Dans cette perspective, l'approche des pratiques managériales en management s'inscrit dans un cadre épistémologique positiviste où la réalité est avant tout connaissable objectivement. Dans cette logique, même les dimensions subjectives ont pour but d'être objectivable. Il convient d'interroger la nature de ce que nous connaissons. Et nous voici devant l'« erreur épistémique » évoquée par Bhaskar (1978), c'est-à-dire la croyance selon laquelle les énoncés sur ce qui existe peuvent toujours être traités comme des énoncés sur la connaissance de ce qui existe.

Le mainstream de la recherche en management s'inscrit dans cette « erreur épistémique »: il y a une grande confusion entre la réalité et ce qui est apparent. De ce point de vue, les méthodologies mises en place ont pour principal objectif de travailler sur des éléments apparents liés aux pratiques managériales. La plupart des travaux publiés dans le domaine du management soulignent les précautions méthodologiques que le chercheur a prises en œuvrant à assurer l'objectivité des informations recueillies en vue d'étoffer 
sa recherche. Rares sont les articles où est évoquée - ou mise en œuvre - une méthode destinée à élaborer des connaissances tirant parti de l'expérience du manager. En procédant de cette façon, les chercheurs privilégient une représentation ontologique de la réalité où le réel est considéré comme une donnée indépendante de l'observateur et antérieure à l'observation (Girod-Séville et Perret, 1999 ; von Glasersfeld, 2000). En ne prenant pas en considération cette possibilité, les chercheurs se privent des connaissances liées à l'expérience irremplaçable que des acteurs liés au management développent dans leurs pratiques. Le recours méthodologique le plus courant de ces chercheurs s'inscrit dans une "réalité de premier ordre », telle que l'appelle Watzlawick (2000). Héritée des sciences classiques, cette réalité de premier ordre correspond avant tout à «l'image de la réalité que nous recevons de nos sens » (Watzlawick, 2000, p. 31). Le travail méthodologique des chercheurs s'apparente, selon l'acception de Lacroux (1999), à une recherche du « vrai ». Ainsi la recherche en management semble-t-elle être avant tout une recherche confirmatoire qui s'intéresse à la validation/réfutation de savoirs existants plutôt qu'à la production de savoirs projetés sur des éléments à venir, destinés à apporter des éclairages sur des préoccupations pour lesquelles il n'existe pas encore de paramètres théoriques satisfaisants, ou permet une théorisation asymétrique qui ne s'inscrit pas dans les logiques théorisantes déjà existantes. Dans cette perspective, il convient de ne pas se limiter au manager et de prendre en considération l'ensemble des parties prenantes qui lui sont liées. Comme le suggèrent Lambin, Gallucci et Sicurello (2009, p. 189), «il faut comprendre le marché comme un écosystème où tous les agents participants sont fonctionnellement connectés ». Il est d'ailleurs étonnant que peu de recherches portent, par exemple, sur les parties prenantes externes des organisations, préférant se limiter à l'organisation elle-même.

De plus, à travers la perspective de l'individualisme méthodologique, les pratiques managériales considèrent avant tout le management comme étant le fait du manager puisqu'il est vu comme son seul organe moteur. Les pratiques managériales n'ont donc pas d'autres substances que celle des managers qui la composent. La compréhension du management sous cet angle met en évidence la filiation avec le réductionnisme cartésien dans lequel il s'est développé depuis l'agir rationnel. Avec le management envisagé comme le produit de l'action humaine individuelle, l'accent est mis sur la prise de conscience des processus mentaux du manager. Cela traduit bien la façon dont la question de l'action managériale a été envisagée : de façon explicite et rationnelle (Barwise, 1989). En se positionnant dans cette logique, les pratiques managériales s'opposent à ce que l'on peut tenir pour acquis en psychologie cognitive, à savoir que les processus mentaux sont pour la plupart inconscients (Sperber, 1997). Pour agir efficacement, un manager doit sélectionner et analyser les conditions de son action. Cette prise de conscience des acteurs d'une situation donnée n'est possible qu'avec des managers dotés d'une certaine capacité de distanciation. Ainsi, il serait possible d'abstraire l'action des circonstances qui l'entourent afin de prendre des décisions optimales. L'introduction de la cognition dans la réflexion sur le management (Lauriol, 1998) n'a pas éloigné pour autant le spectre du manager rationnel évoqué précédemment. Au final, quelle que soit la sophistication apportée par les réflexions autour de la cognition managériale les biais cognitifs et 
les styles cognitifs, elles ne permettent pas de s'affranchir de l'individualisme méthodologique, bien au contraire. Dans la pratique, les outils du management apparaissent aujourd'hui comme le symbole de la domination de l'individualisme méthodologique pour aborder le management. Bien souvent, ces outils peuvent être assimilés à une forme de réductionnisme suggérant que le management peut s'expliquer par la juxtaposition de divers thèmes (marché, ressources humaines, financier...).

Ainsi, pour réussir, il suffirait d'optimiser les différents paramètres mis en évidence par les réflexions dans le domaine du management. Encore une fois, quelle que soit l'orientation prise, les différentes orientations par rapport aux pratiques managériales ont montré leurs limites. Aujourd'hui, nous voyons bien les limites qui incitent à envisager le management autrement. Il conviendrait de ne pas agir comme l'ivrogne qui s'obstine à ne chercher ses clés que sous le lampadaire, car c'est le seul endroit qui soit éclairé. Nous devons transformer notre façon de voir les choses.

\section{LES SITUATIONS DE GESTION POUR COMPRENDRE LA COMPLEXITÉ DES PRATIQUES MANAGÉRIALES}

Lisa est manager dans un cabinet de consultant. Elle est responsable du service Community management. Elle se pose des questions sur le fonctionnement actuel de son service par rapport aux objectifs de l'organisation. Mathias est manager dans une entreprise d'agroalimentaire. Il cherche à valoriser les pertes matières liées au processus de fabrication. Autour de la table, il y a des personnes de l'équipe de production et des personnes d'une autre entreprise intéressée à valoriser leurs déchets. Sandra est ingénieure informatique dans une entreprise qui développe des applications. Elle a besoin de s'entourer de nouvelles compétences. Pour les recruter, elle est amenée à rencontrer différentes personnes. Voilà quelques exemples de ce qu'on peut qualifier de situation de gestion.

À première vue, un triptyque ressort de ces situations : le manager, l'objet de gestion lou artefact) et le contexte. Voici ce qui caractérise ces situations : le manager est amené à agir dans un contexte où il est en interaction avec un objet de gestion (fonctionnement d'un service, pertes matières, recrutement de nouvelles compétences) et un contexte comme d'autres personnes, une stratégie, une réglementation... par rapport à une intentionnalité. L'intérêt de la recherche se déplace pour aller des pratiques managériales vers l'intentionnalité, de la face visible de l'iceberg à sa face cachée. Cette évolution caractérise notamment la volonté de s'affranchir de l'agir rationnel et de l'agir normatif. Il convient donc dans la recherche en management d'aller au-delà de la notion de pratiques managériales et de s'emparer de cette notion d'intentionnalité pour faire avancer la recherche. Nous sommes dans la globalité du manager, c'est-à-dire le manager, son objet de gestion, son contexte et son interaction avec son organisation et son contexte. Dans cette perspective, le 
regroupement de ces différents éléments : l'intentionnalité, le contexte, l'objet de gestion et leurs interactions, forme ce qu'il est convenu d'appeler une situation de gestion. Nous proposons d'utiliser cette grille d'analyse pour aborder la complexité de l'action dans les organisations, c'est-à-dire selon l'optique de l'agir entrepreneurial (Schmitt, 2015). Ainsi, pour nous, la situation de gestion devient l'unité d'analyse qu'il conviendrait de mobiliser pour aborder les pratiques entrepreneuriales. Cette unité d'analyse peut s'appliquer à différents cas comme une administration publique (Schmitt et al., 2011), l'entrepreneuriat (Schmitt, 2009).

\section{L'intentionnalité comme ferment des situations de gestion}

Dans cette notion de situation de gestion, deux caractéristiques sont à mettre en exergue. La première caractéristique renvoie à la relation au temps. En effet, dans une logique d'intentionnalité, le futur est susceptible de jouer un rôle important dans le développement des actions présentes (Schmitt, 2012a). Dans cette perspective, l'objet de gestion apparaît comme étant empreint d'intentionnalité pour l'action présente et à venir. Par voie de conséquence, la seconde caractéristique renvoie à la notion de problématisation, laquelle consiste à donner un sens aux idées portées par les managers afin de relier le futur et le présent.

Une caractéristique des situations de gestion correspond à l'approche temporelle (Urban et Zuchella, 2011). Du point de vue temporel, la particularité d'une situation de gestion réside dans le lien que l'on peut établir entre la situation actuelle et la situation souhaitée et donc future ${ }^{3}$. Derrière ce lien se trouve la notion de conception développée par Simon (1996), qui résulte de la capacité d'imaginer des dispositions visant à changer une situation existante en une situation préférée. La situation de gestion est formée par l'ensemble des circonstances dans lesquelles le manager se trouve et où sont reliés le présent et le futur (Cossette, 2001). Elle apparaît comme la façon dont le manager expérimente le réel. L'expérience du réel va se faire «chemin faisant » par rapport à cette intentionnalité, qui est à comprendre non selon l'acception de Shapero et Sokol (1982), Bird (1988) ou encore de Krueger et Carsrud (1993), qui relève de l'agir normatif, mais selon l'acception phénoménologique (Berglund, 2007 ; Schmitt, 2015). L'intentionnalité désigne la capacité qu'a un individu de se forger des représentations portant son empreinte ldésir, volonté, rapport au monde...) à l'instar des actions qu'il entreprend. Nous passons donc de la simple conscience de l'action par le manager à une prise de conscience de cette action. L'action permettant de relier le futur souhaité et le présent est dite intentionnelle, car elle exprime le sens que le manager attribue aux choses. Souvent les travaux portant sur le management envisagent implicitement une relation entre le présent et le futur de façon chronologique et linéaire. Cette façon d'aborder la temporalité provient d'une approche

3. Ce futur souhaité peut trouver d'autres échos dans la littérature. En effet, un lien peut être fait ici avec les réflexions de Keynes pour qui ce n'est pas le profit obtenu qui guide ou incite à investir, mais le profit espéré. 
conventionnelle du temps allant du présent au futur et n'est pas sans affecter l'action des managers. Nous retrouvons ici l'idée émise par Watzlawick (1988, p. 74) et soulignée dans un contexte différent : « Ce n'est pas le passé, mais le futur, qui détermine le présent ». Par conséquent, il n'y a pas de management sans intentionnalité du manager.

\section{Quand manager, c'est problématiser}

Adoptant une perspective rationnelle, les recherches en management considèrent les situations de gestion de manière ontologique, c'est-à-dire de façon objective, les tenant pour une réalité existant en dehors de la personne qui évolue dans la situation concernée. Dans cette logique, comprendre une situation de gestion reviendrait seulement à pouvoir l'observer. Il est possible dans un paradigme épistémologique constructiviste téléologique (Avenier, 2007) de considérer une situation de gestion « comme la mise en ordre et l'organisation d'un monde constitué par nos expériences » (Glasersfeld, 1984, p. 27). De même, une situation de gestion peut être considérée comme une construction humaine ayant une intentionnalité et tenant compte du contexte, à un moment donné, de l'objet de gestion y afférent par rapport à un futur projeté (Sarasvathy, 2001). Pour la recherche en management, il s'agit d'amener les managers à construire un écosystème constitué de leurs parties prenantes afin de conforter leur prise de conscience de la situation de gestion et de l'intentionnalité à l'œuvre dans le soubassement de cette situation. Alors que les approches dans le domaine du management se sont souvent affranchies de la relation entre fin et moyen, il semble important d'intégrer cette dimension pour comprendre les situations de gestion. En effet, la dialectique fins-moyens joue un rôle essentiel dans l'articulation récursive du contexte du moment et de celui du futur.

La mise en ordre évoquée précédemment est un élément important de la situation de gestion. Il s'agit pour le manager de construire du sens, pour reprendre la terminologie employée par Weick (1999), ou encore de construire un problème, selon Dewey (1938) à partir des actions menées « chemin faisant » par le manager. Le manager crée donc un écosystème que l'on peut appeler situation de gestion. L'intérêt de recourir aux situations de gestion pour comprendre le management consiste notamment à sortir de la logique centrée sur le pouvoir et notamment le pouvoir hiérarchique pour amener une lecture de la complexité des situations envisagées. La situation apparaît alors comme un processus néguentropique consistant à chercher à organiser les choses par rapport à un désordre perçu et, par conséquent, à leur donner du sens (Schmitt, 2000). On retrouve cela dans la vision construite par le manager qui cherche à donner du sens à son présent par la projection d'un futur possible, tant pour lui que pour les parties prenantes de la situation de gestion. Cette vision n'est finalement rien d'autre que la représentation de la situation de gestion par le manager et les parties prenantes de la situation. Des questions sous-jacentes émergent de cette remarque : quels sont les mécanismes en jeu permettant la construction de sens ? Comment permettre au manager de construire du sens ? Comment établir l'intentionnalité d'une situation de gestion? L'une des réponses pouvant y être apportées 
renvoie aux mécanismes de construction d'un « espace de problématisation », appelé aussi « espace de problème » par Simon (1996) ou encore Spielraum par Heidegger $(1964)^{4}$. Cet espace de problématisation, permet de faire le lien entre les relations suivantes au sein d'une situation de gestion :

- contexte futur et contexte actuel,

- fins-moyens.

Les situations de gestion peuvent s'envisager comme une situation dite ouverte, c'est-à-dire partant d'une solution perçue par le manager, centrée sur une intentionnalité pour arriver à une solution plus contextualisée. Cette transformation est ce qu'il convient d'appeler l'espace de problématisation et correspond à la construction d'un objet de gestion. Cet objet de gestion peut être vu comme un artefact, jouant le rôle d'intermédiation entre le manager et les parties prenantes de la situation de gestion.

De ce point de vue, nous rejoignons les différents auteurs (Hatchuel, 2000 ; Micaëlli et Forest, 2003), qui considèrent le management comme faisant partie des sciences de l'artificiel au sens de Simon (1996). L'espace de problématisation constitue d'une certaine manière l'espace dans lequel le manager va être amené à construire sa représentation de la situation de gestion que lui-même va régulièrement tester par rapport aux parties prenantes de la situation. En d'autres termes, en situation, le manager doit se poser différentes questions et développer un certain nombre d'hypothèses plausibles pour construire une représentation cohérente et robuste de la situation de gestion. Cette représentation de la situation n'est pas figée ni linéaire, bien au contraire, elle évolue en fonction des actions de confrontation, formelles ou non. Le manager fait l'expérience d'une situation de gestion à travers l'action dès le début pour faire évoluer sa construction de sens à partir d'une intentionnalité de départ. La mise en situation du manager permet de trouver des réponses à ses questions et de faire émerger des questions qui n'auraient pas vu le jour sans cette mise en situation. En agissant ainsi, les managers sont amenés à faire évoluer la situation de gestion dans laquelle ils se trouvent à travers la logique de problématisation. Toutefois, à ce stade de la réflexion, force est de constater le peu d'importance accordée aux outils consacrés à la problématisation, par rapport à l'abondance de ceux voués à la résolution de problèmes. Or, problématiser ne s'improvise pas. Ces divers constats montrent qu'une grande partie des difficultés rencontrées par les managers est plus liée à la capacité à problématiser, c'est-à-dire à développer une expérience par rapport à la complexité vécue par le manager, qu'à la capacité à résoudre des problèmes.

\section{Définition et avenues de la recherche}

Une situation de gestion pourrait se définir comme l'ensemble des événements, des circonstances, des relations concrètes au milieu desquels se trouvent un ou des managers. Cette situation n'est pas donnée, elle est construite par le manager en action en fonction de son intentionnalité et du futur souhaité. La

4. Traduction littérale de l'allemand : espace pour manœuvrer. Ainsi, le Spielraum désigne le champ d'action, le champ opérationnel effectif d'une personne en situation, appelé aussi le champ des possibles. 
situation est évolutive dans le temps. Une situation de gestion est vue comme une construction ciblée, façonnée par le manager et le fruit d'une relation que le sujet entretient avec le monde par ses actes. Au travers de cette relation, le sujet se construit, construit des artefacts, construit du sens et participe à la construction d'autrui en relation avec la situation. La relation à autrui est une notion importante dans la littérature en matière de situations (Suchman, 1987). Comme toute personne, le manager ne peut se dissocier de lui-même, ni de la situation, ni de son action en situation. La notion de situation de gestion fait émerger à la fois au regard du manager, l'objet de gestion et le contexte reliés dans une action intentionnelle (Bird, 1988). Le sens n'est donc pas dans les situations ; il est introduit par des actes d'interprétation au fur et à mesure de l'évolution de la situation de gestion. La notion de situation de gestion peut apparaître comme un maillon manquant dans la compréhension de la complexité des pratiques managériales. Elle s'inscrit dans ce que Venkataraman et al. (2012) appellent le développement, «a new nexus around actions and interactions ». En effet, la représentation de la situation agit comme un « espace de problématisation » entre le contexte dans lequel le manager évolue à partir d'une finalité, qui elle-même peut évoluer en fonction du contexte, et un objet de gestion, qui serait le point de départ de la situation de gestion.

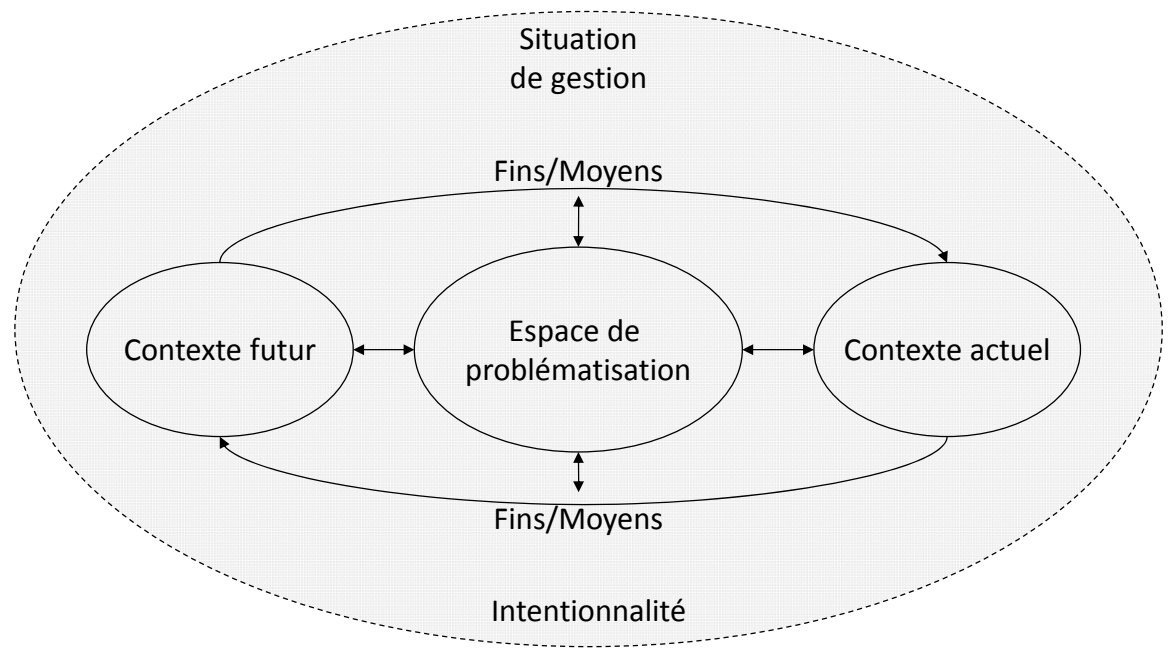

Figure 1. L'articulation des composants d'une situation de gestion

L'action du manager ne peut être envisagée simplement comme une réponse à une situation donnée, dans une logique de stimuli-réponse. Les actions mises en œuvre par le manager vont dépendre de la façon dont il conceptualise la situation dans laquelle il se trouve, c'est-à-dire la façon dont il va donner du sens à la situation en fonction d'une intentionnalité initiale. Les actions vont fortement dépendre de la construction de sens faite par le manager en situation, donc de la délimitation de l'espace de problématisation. En s'inscrivant dans le prolongement des sciences de l'artificiel (Venkataraman et al., 2012 ; Schmitt, 2015), la notion de situation de gestion permet de dégager des voies de recherche intéressantes. 
En ce qui concerne le type de rationalité en jeu dans les situations de gestion, il ne s'agit pas de développer des rationalités substantielles portant sur le contenu et engendrant des outils à utiliser dans une situation déterminée. L'objectif est de développer des rationalités procédurales (Simon, 1969) plus aptes à modifier la manière d'agir au sens effectual du terme (Sarasvathy, 2001). Cela renvoie à l'expérience vécue par le manager. Cette rationalité procédurale est d'autant plus pertinente dans le domaine du management que, comme évoqué précédemment, les situations rencontrées par les managers sont des situations ouvertes, où il n'y a pas de solutions prédéterminées et encore moins de solutions optimales. Dans cette perspective, le management est à rattacher à d'autres domaines en lien avec la notion de situation. Ainsi, à travers les situations en général et les situations de gestion en particulier, il s'agit avant tout de s'intéresser à l'agir humain en situation. Des passerelles nouvelles et stimulantes s'offrent au management pour se comprendre et de façon réciproque le management peut apporter un éclairage singulier aux situations dans d'autres domaines. Mobiliser la notion de situation en management, c'est considérer cette notion comme une grille de lecture pertinente pour aborder la complexité des organisations avec une méthode de raisonnement à part entière. Dans cette perspective, les situations de gestion ne sont plus envisagées comme des situations singulières mais comme des situations ordinaires. La réflexion sur les situations de gestion nous enseigne que les situations évoquées sont plus répandues que l'on ne croit. On retrouve ici le lien avec les travaux de Simon sur la conception (1996) et avec les travaux de Joas sur l'agir créatif (1996). À travers la rationalité procédurale, il s'agit bien de chercher le sens de l'expérience vécue par le manager en situation. Pour aborder le sens de cette expérience, il est essentiel de s'appuyer sur le triptyque qui compose une situation de gestion évoquée en amont : le manager, l'objet de gestion et le contexte. Le recours à l'expérience vécue par le manager en situation inscrit encore plus le management dans une perspective phénoménologique. Des perspectives nouvelles en matière de méthodologie s'ouvrent ainsi aux chercheurs en management (Berglund, 2007 ; Schmitt, 2015). Il s'agit de compléter les méthodes de recherches actuelles qui utilisent notamment des questionnaires, des discours et des échelles a posteriori avec des méthodes s'inscrivant dans le cours de l'action pour en comprendre le sens. Nous pensons ici principalement au développement de recherches-action qui vont se focaliser sur les situations de gestion en cours (Avenier et Schmitt, 2010 ; Schmitt et Filion, 2009).

De cette rationalité procédurale, l'expérience vécue par le manager engendre dans un premier temps la construction d'hypothèses, qui peuvent être qualifiées de plausibles pour le manager au sens d'Avenier (2007). Pour guider son action et construire sa vision de la situation, le manager construit une représentation d'une situation à partir de son intentionnalité qu'il organise autour d'un jeu d'hypothèses plausibles et cela par rapport au contexte dans lequel il se projette. Problématiser, c'est alors construire des hypothèses plausibles à partir de cette intentionnalité qui fournissent des repères heuristiques destinés à éclairer l'entrepreneur dans sa réflexion et son action. La validation ou non de ces hypothèses plausibles amène à « tester » la robustesse de l'espace de problématisation. 
Autres notions qu'il convient d'explorer: la robustesse de la représentation d'une situation de gestion et la cohérence de la situation de gestion. Cela revient à intégrer la représentation de la situation de gestion dans un contexte présent pour voir sa validité par rapport aux représentations des parties prenantes. À travers les situations de gestion, il s'agit bien de favoriser la confrontation des représentations des parties prenantes par rapport au « bricolage » dont les managers font souvent preuve (Tomasino, 2007). Les situations de gestion évoluent en fonction de ces confrontations et de la robustesse des représentations des acteurs de la situation. Par son action, le manager est amené à « tester » la robustesse de sa représentation. Ce pan de la littérature reste encore largement absent de la recherche en management. Autre notion à développer au niveau du management : la cohérence de la situation de gestion. Trop souvent l'évaluation d'une situation de gestion porte sur les résultats d'une action, mais rarement sur sa cohérence. Étant donné que la situation de gestion est un construit humain à partir de son environnement, il ne s'agit pas d'évaluer ce construit, mais sa cohérence. Sinon, le risque est grand de brider le manager dans sa façon de faire. Comme la situation de gestion s'inscrit dans une logique cognitive, il est important de développer des systèmes d'évaluation appropriés autour de cette notion de cohérence. Trop souvent les systèmes d'évaluation cherchent à évaluer les constituants d'une approche rationnelle (Sarasvathy, 2001).

Derrière ces différentes notions évoquées, il s'agit bien d'enrichir le débat portant sur les pratiques managériales qui structure la recherche en management. Notre contribution dans le domaine s'inscrit principalement dans une perspective de construction de la réalité par le manager (Glasersfeld, 1984). Dans cette perspective, cela signifie que le manager construit un sens en imposant un ordre sur le monde qui l'entoure à partir de son intentionnalité.

\section{CONCLUSION}

Dans cet article, l'objectif visé était de mettre en lumière une notion peu mobilisée dans la recherche en management : les situations de gestion. La première partie a permis de comprendre l'absence de cette notion en management alors qu'elle est très présente dans d'autres domaines des sciences humaines. En effet, cette posture peut se comprendre notamment à la lumière d'un cadre épistémologique dominant autour du positivisme où la connaissance générée est avant tout descriptive dans le but de comprendre la réalité. Pour cela, nous avons esquissé, à partir des études en management, la place de cette notion. Même si des traces peuvent être envisagées, les situations de gestion ne sont pas présentes dans la recherche dans le domaine. En partant de la définition proposée par Girin (1990), nous avons cherché à montrer son intérêt pour la recherche en management. Pour cela, nous avons dû étayer nos propos par des travaux de recherche portant sur les situations en général.

La seconde partie s'est alors attachée à l'identification des caractéristiques de ces situations de management pour appréhender l'expérience vécue par les managers. Le premier point s'est attaché à montrer l'importance de 
l'intentionnalité pour comprendre les situations de gestion. Le deuxième point a intégré une autre notion : l'espace de problématisation. Cet espace correspond aux mécanismes permettant de faire le lien entre l'intentionnalité, le présent et le futur. À travers cet espace, l'intentionnalité donne du sens à la situation de gestion, le futur conditionne le présent et le présent influe sur le futur. Cet espace est délimité par des contraintes et des opportunités ${ }^{5}$. Le troisième point propose une définition d'une situation de gestion et les avenues de recherche qui sont liées.

Finalement, le parti pris de cet article, en l'occurrence celui d'envisager la recherche en management à travers la notion de situation de gestion, ne doit pas être compris comme un rejet des recherches dans le domaine. Il est à entendre comme mettant en évidence une opportunité pour la recherche en management de s'enrichir en s'autorisant à ne plus se limiter seulement à des recherches s'inscrivant dans une hypothèse implicite de séparation entre l'auteur de l'action et les résultats de l'action - lesquelles sont en fait indissociablement liées au sein du management comme le sont l'avers et le revers d'une pièce. Cet article se veut aussi un plaidoyer pour le développement de recherches portant sur l'expérience vécue par les managers afin d'envisager le management dans sa globalité et ses interactions (Schmitt, 2008). Il s'agit de développer des représentations holistiques et dynamiques du management (Bygrave et Hofer, 1991). Ce type de recherches ne s'inscrit pas, comme le souligne Von Foerster (2000), dans la volonté de savoir ce que nous connaissons mais plutôt dans la volonté de savoir comment nous connaissons.

\section{BIBLIOGRAPHIE}

Avenier, M.-J. (2007), « Repères pour la transformation d'expérience en science avec conscience », dans Avenier M.-J. et C. Schmitt (dirs.), La Construction de Savoirs pour l'Action, L'Harmattan, pp. 140-170.

Avenier, M.-J. et Schmitt, C. (2010), «Et si c’était l’expérience du réel, plutôt que le réel luimême, qui était connaissable : quelles conséquences pour la recherche en entrepreneuriat ?», communication présentée au Colloque Cifepme, octobre, Bordeaux.

Barwise, J. (1989), The Situation in Logic, Stanford, Center of the Study of Language Information. Béguin, P., Clot, Y., « L'action située dans le développement de l'actualité », Revue activités, vol 1, $\mathrm{n}^{\circ} 2$, octobre 2004, p.27-49.

Berglund, H. (2007), «Researching entrepreneurship as lived experience», dans Neergaard, $H$. et Ulhøi, J. (dir.), Handbook of Qualitative Research Methods in Entrepreneurship, Cheltenham, Edward Elgar, p. 75-93.

Bhaskar, R. (1978), A Realist Theory of Science, Brighton, Harvester Press.

Bird, B.J. (1988), «Implementing entrepreneurial ideas: The case for intention», Academy of Management Review, vol.13, n³, p. 442-453.

Burrell, G. et Morgan, G. (1979), Sociological Paradigms and Organizational Analysis: Elements of the Sociology of Corporate Life, Londres : Heinemann.

Popper, K. (1997), Toute vie est résolution de problèmes, Paris, Actes Sud.

Bygrave, W.D. et Hofer, C.W. (1991), «Theorizing about entrepreneurship», Entrepreneurship Theory and Practice, vol. 16, n², p. 13-23.

5. À ce sujet, les travaux portant sur les opportunités n'intègrent quasiment pas les contraintes, alors que les deux semblent indissociables. 
Dewey, J. (1938), Logic: The Theory of Inquiry, New York, Holt, Rinehart and Winston.

Foerster, H., von (2000), «Éthique et cybernétique de second ordre», dans Watzlawick, P. et Nardone, G. (dir.), Stratégie de la thérapie brève, Paris, Seuil, p. 60-76.

Fornel, M., de et Quéré, L. (1999), La logique des situations, nouveaux regards sur l'écologie des activités sociales, Paris, Éditions de l'École des hautes études en sciences sociales.

Girin, J., «L'analyse empirique des situations de gestion : éléments de théorie et de méthodes », dans A.-C. Martinet (coordination), Epistémologies et Sciences de Gestion, Editions Economica, Paris, 1990, p. 141-181.

Girin, J. (2011), «Empirical analysis of management situations: Elements of theory and method», European Management Review, vol. 8, p. 197-212.

Girod-Séville, M. et Perret, V. (1999), «Fondements épistémologiques de la recherche», dans Thiétart, R.A. (dir.), Méthodes de recherche en management, Paris, Dunod, p. 13-33.

Glasersfeld, E., Von (1984), «An introduction to radical constructivism», dans Watzlawick, P. (dir.), The Invented Reality, New York, Norton, p. 17-40.

Glasersfeld, E., Von (2000), «La construction de la connaissance», dans Watzlawick, P. et Narbonne, G. (dir), Stratégie de la thérapie brève, Paris, Seuil, p. 34-48.

Hatchuel, A. (2000), «Quel horizon pour les sciences de gestion? Vers une théorie de l'action collective », dans David, A., Hatchuel, A. et Laufer, R. (dir.), Les nouvelles fondations des sciences de gestion, Paris, Vuibert/Fnege, p. 7-43.

Hatchuel, A. (2005), « Pour une épistémologie de l'action. L'expérience des sciences de gestion », dans Teulier R. et Lorino P., Entre connaissances et organisation: l'activité collective, La découverte, Paris, pp. 73-92.

Heidegger, M. (1964), L'être et le temps, Paris, Gallimard.

Joas, H. (1996), The Creativity of Action, Chicago, Chicago University press.

Journé, B., Raulet-Croset, N. (2008), « Le concept de situation : contribution à l'analyse de l'activité managériale dans un contexte d'ambiguïté et d'incertitude », Revue Mandagement, vol. 11, $n^{\circ} 1$, p. 27-55.

Krueger, N.F. et Carsrud, A.L. (1993), «Entrepreneurial intentions: Applying theory of planned behavior», Entrepreneurship and Regional Development, vol. 5, p. 315-330.

Lacroux, F. (1999), «La modélisation dans le contrôle de gestion », dans Dupuy, Y. (dir.), Faire de la recherche en contrôle de gestion ?, Paris, Vuibert-Fnege, p. 21-29.

Lambin, J.-J., Gallucci, C., et Sicurello, C. (2009), Dirección de marketing: gestión estratégica y operativa del mercado, Mexico, McGraw-Hill.

Lauriol, J. (1198), La décision stratégique en action, une perspective socio-cognitive, L'Harmattan, Paris.

Lawson, T. (1996), Economics and Reality, Londres, Routledge.

Lorino, P. (2005), « Théories des organisations, sens et action », dans Lorino, P. et Teulier, R. (dir.), Entre connaissance et organisation : l'activité collective, Paris, La Découverte, p. 54-71.

Micaëlli, J.-P., Forest, J. (2003), Artificialisme, introduction à une théorie de la conception. Presses polytechniques et universitaires romandes.

Popper, K. (1991), La connaissance objective, Paris, Flammarion.

Sarasvathy, S. (2001), «Causation and effectuation: Toward a theoretical shift from economic inevitability to entrepreneurial contingency», Academy of Management Review, vol. 26, $n^{\circ} 2$, p. 243-263.

Schmitt C., Fabbri R., Gallais M. (2011), Situation de gestion, proximité cognitive et changement dans les organisations, Revue Française de Gestion, $n^{\circ} 213$, p. 157 à 169.

Schmitt, C. (2000), «Ordre et désordre en PME: contribution du visuel au développement organisationnel », Direction et gestion, $n^{\circ} 180-181$, p. 45-60.

Schmitt, C. (2008), «Éléments de convergence et de perspectives pour une approche anthropocentrée des pratiques entrepreneuriales», dans Schmitt, C. (dir.), Regards sur l'évolution des pratiques entrepreneuriales, Québec, Presses de l'Université du Québec, coll. « Entrepreneuriat et PME », p. 319-330.

Schmitt, C. (2009), « Les situations entrepreneuriales : proposition d'une nouvelle grille d'analyse pour aborder le phénomène entrepreneurial », Revue économie et sociale, $n^{\circ} 3$, p. 11-25. 
Schmitt, C. (2012), «Créer son futur pour agir au présent», dans Filion, L.J., Ananou, C. et Schmitt, C. (dir.), Réussir sa création d'entreprise sans business plan, Paris, Editions Eyrolles, p. 69-83.

Schmitt, C. (2015), L'agir entrepreneurial, repenser l'action des entrepreneurs, Presses de l'Université du Québec, Québec.

Schmitt, C. et Filion, L.J. (2009), « Le réel expérimenté pour explorer la TPE : apports conceptuels et méthodologiques », Management \& Avenir, no 30, p. 207-226.

Shapero, A. et Sokol, L. (1982), «The social dimensions of entrepreneurship», dans Kent, C. et al. (dir.), The Encyclopedia of Entrepreneurship, Englewood Cliffs, Prentice-Hall, p. 72-90.

Simon, H.A. (1996), The Sciences of the Artificial, Cambridge, MIT Press.

Sperber, D. (1997), « Individualisme méthodologique et cognitivisme », dans Boudon, R., Chazel, F. et Bouvier, A. (dir.), Cognition et sciences sociales, Paris, Presses universitaires de France, p. 123-136.

Suchman, L.A. (1987), Plans and Situated Actions: The Problem of Human-Machine Communications, Cambridge, Cambridge University Press.

Tomasino, D. (2007), «The psycho-physiological basis of creativity and intuition: Accessing "the zone" of entrepreneurship», International Journal of Entrepreneurship and Small Business, vol. 4, $n^{\circ} 5$, p. 528-542.

Urban, S. et Zuchella, A. (2011), «Building the future through real value creation and innovation: Achieving competitiveness in a chaotic world», International Journal of Entrepreneurship and Small Business, vol. 13, n², p. 126-149.

Van de Ven A. H. (2007), 'Engaged scholarship: A guide for organizational and social research', Oxford University Press.

Venkataraman, S., Sarasvathy, S. D., Dew, N. et Forster, W. (2012), «Whither the promise? Moving forward with entrepreneurship as a science of the artificial», Academy of Management Review, vol. 37, n 1, p. 21-33.

Watzlawick, P. (1988), «Effet ou cause ?», dans Watzlawick, P. (dir.), L'invention de la réalité, contribution au constructivisme, Paris, Seuil, p. 73-78.

Watzlawick, P. (2000), « La construction des réalités cliniques», dans Watzlawick, P. et Narbonne, G. (dir.), Stratégie de la thérapie brève, Paris, Seuil, p. 19-33.

Weick, K.E. (1999), «Theory construction as disciplined reflexivity: Tradeoffs in the 90's», Academy of Management Review, vol. 24, n4, p. 797-806.

Christophe SCHMITT est Vice-Président de l'Université de Lorraine. Il est Professeur des Universités à l'IAE de Metz (France). Il y est Titulaire de la Chaire « Entreprendre » et responsable du Pôle entrepreneuriat étudiant de Lorraine (PeeL). Il est également Professeur associé à la Louvain School of Management (Belgique) et à la Haute Ecole de Gestion de Fribourg (Suisse). Ses articles et ouvrages portent sur le développement d'une théorie de l'action entrepreneuriale. Son dernier ouvrage s'intitule « La fabrique de l'entrepreneuriat » et est paru aux éditions Dunod. 